\title{
Algunos elementos para comprender el Brexit y su impacto en las teorías del regionalismo
}

\author{
Some elements to understand Brexit and its impact on the theories of \\ regionalism
}

Nicolás Pose $e^{1}$

\section{Resumen}

El 23 de junio de 2016 los británicos decidieron, mediante referéndum, abandonar la Unión Europea. Este artículo busca aportar algunos elementos para responder dos preguntas relacionadas: 1 - ¿Cuáles fueron los factores que condujeron al Brexit?; 2- ¿Cómo impacta este fenómeno en las teorías sobre la conformación de acuerdos regionales? Para abordar el primer punto, se discute el rol de la representación de intereses en la convocatoria al referéndum, y posteriormente se analiza la construcción de dichos intereses desde la literatura que destaca el rol de las ideas. Mientras que sobre el segundo punto, se aborda el cuestionamiento que dicho evento supone para los planteos que sugieren un camino unidireccional hacia la conformación de acuerdos regionales, y por el contrario se enfatiza en la necesidad de contar con nuevos instrumentos teóricos que den cuenta de los resultados mixtos en términos de integración y desintegración que actualmente se observan en la economía mundial.

Palabras claves: Brexit, Unión Europea, regionalismo, representación de intereses - ideas

Recibido: 23 de agosto de 2019 Aceptado: 26 de noviembre de 2019 Publicado: 20 de diciembre de 2019

${ }^{1}$ Doctorando en Ciencia Política, Universidad de la República, Uruguay. Magíster en Economía Política Internacional, London School of Economics and Political Science, Reino Unido. Diplomado en Estudios Internacionales y en Economía, y Licenciado en Ciencia Política, Universidad de la República. Docente e investigador del Programa de Estudios Internacionales y del Departamento de Ciencia Política, Universidad de la República, Montevideo, Uruguay. Correo electrónico: nicolas.pose@cienciassociales.edu.uy 


\section{Abstract}

On June $23^{\text {rd }} 2016$, the British decided, through a referendum, to abandon the European Union. This paper seeks to provide elements to answer to related questions: 1- Which were the factors that drove Brexit? 2- How does this phenomenon affect the theories that explain the conformation of regional agreements? To approach the first question, the article discusses the role of interest representation in the calling of the referendum, and then analyses the construction of those interests through the role of ideas. Secondly, it examines how this event questions the approaches that suggest a unidirectional path to the conformation of regional agreements, and on the contrary, it emphasises the need of developing new theoretical instruments to explain the mixed results in terms of integration and disintegration that we currently observe in the global economy.

Keywords: Brexit, European Union, regionalism, interest representation- ideas

\section{Introducción}

La decisión del Reino Unido de salir de la Unión Europea (UE), conocida popularmente como "Brexit", fue durante buena parte de 2016, al menos hasta la elección de Donald Trump en Estados Unidos, la principal sorpresa de la política mundial. Aunque los sondeos de opinión venían anunciando que el referéndum convocado para decidir el futuro de la relación entre las Islas Británicas y el Continente tendría un resultado sumamente ajustado -ver YouGov (2016)- líderes mundiales, analistas e inversores apostaron, en una suerte de wishful thinking colectivo, por la opción de que la permanencia triunfaría. Sin embargo, el 51,9\% de los británicos que acudieron a las urnas optaron por la salida.

Este artículo busca proponer algunos elementos para abordar dos preguntas relacionadas. La primera de ellas se refiere a cuáles fueron los factores que condujeron al Brexit, para lo que se debe dar cuenta de dos fenómenos vinculados. En primer lugar, es necesario abordar la convocatoria a un referéndum por parte del entonces primer ministro David Cameron, para lo cual se recurre a las teorías de representación de intereses (Schonhardt-Bailey, 2006), y se presenta evidencia cuantitativa que apoya esta lógica. Y en segundo lugar, es preciso considerar la construcción de dichos intereses, para lo que se observa que los modelos estándares de derivación de preferencias sintetizados en el enfoque open economy politics (OEP) (Lake, 2009) no dan una respuesta adecuada, por lo que se recurre a propuestas 
alternativas basadas en el rol de las ideas y las narrativas (Blyth, 2002; Woll, 2008; Rodrik, 2014).

La segunda pregunta relacionada que se aborda refiere al impacto del Brexit como fenómeno para las teorías que buscan explicar la conformación de acuerdos regionales. En particular, se identifica que el Brexit, como clase de un conjunto de fenómenos de desintegración económica de emergencia reciente, desafía a las teorías de "difusión competitiva" del regionalismo (ej. Baldwin, 1993, 1997; Baccini y Dür, 2012), las que, producto de las dinámicas de competencia que fomentan los acuerdos preferenciales de comercio, esperan una profundización unidireccional de la tendencia hacia su conformación. Y como contrapartida, se esbozan algunas ideas basadas en la tensión entre globalización, democracia y Estado, siguiendo el esquema planteado por Rodrik (2011), con el objetivo de dar cuenta de la evidencia mixta entre integración y desintegración que actualmente se observa en las relaciones económicas internacionales.

El trabajo se estructura de la siguiente manera. La siguiente sección busca aportar elementos para responder la primera pregunta planteada. Para ello, en primer lugar analiza los aportes de la lógica de la representación de intereses, y luego presenta el ejercicio empírico que respalda su relevancia. Tras esto, considera la construcción del interés sobre el Brexit, mediante el análisis de las ideas y las narrativas que lo delinearon. La tercera sección se aboca a responder la segunda pregunta planteada, para lo que analiza el impacto del Brexit sobre las teorías de conformación competitiva de acuerdos regionales, potenciales alternativas, y el efecto sobre el propio proceso de integración europeo. Finalmente, se presentan las conclusiones.

\section{Representación y construcción de intereses en el Brexit}

El Brexit fue consecuencia directa del resultado de un referéndum, en donde la ciudadanía se expresó de forma directa sobre la relación del Reino Unido con la UE, sin mediación de los representantes políticos. Sin embargo, este trabajo argumenta que la representación de intereses es un elemento clave para entender este fenómeno, pues la convocatoria a un referéndum sobre el tema no se explica sin su consideración. Las teorías de representación de intereses plantean que los políticos toman decisiones sobre políticas públicas de forma tal de representar los intereses de sus votantes (los que en general se entienden como intereses materiales), pues su objetivo es acceder y/o mantenerse en cargos electivos, y para ello necesitan del apoyo, financiero y electoral, de los grupos y sectores sociales que representan. Y dado que las políticas de relacionamiento o integración con la economía mundial 
tienen fuertes implicancias distributivas, la representación de intereses tiene particular relevancia para explicar las conductas de los representantes políticos en este campo (Milner y Judkins, 2004; Schonhardt-Bailey, 2006; Hiscox, 2014). Sobre dicha lógica se construye el primer argumento planteado por este trabajo, que sostiene que el referéndum fue convocado, al menos en parte, por una lógica de representación de intereses.

El Reino Unido ingresó en la Comunidad Económica Europea bajo un gobierno conservador en 1973, y la decisión fue refrendada en 1975 por la ciudadanía mediante una consulta directa. Sin embargo, durante la década de 1980, bajo el gobierno de Margaret Thatcher, comenzaron a registrarse algunos cuestionamientos a la Comunidad producto de los requerimientos de contribuciones británicas al presupuesto comunitario, que fueron alimentando un creciente sentimiento euroescéptico dentro del Partido Conservador. Este sector del partido creció paulatinamente en número y, en 1993, se plasmó en la rebelión de 40 diputados frente a su propio gobierno respecto a la relación con Europa. De todos modos, no fue hasta 2011 que dicho movimiento alcanzó dimensiones significativas. Ese año, 81 diputados tories sin cargos gubernamentales desafiaron al gobierno de Cameron en una votación para llamar a un referéndum que planteara la opción de retiraste de la UE, impulsada por una petición pública que había alcanzado más de 100.000 firmas (BBC, 2011). En este contexto, Cameron incluyó en su plataforma hacia las elecciones generales de 2015, en la que renovó su mandato y esta vez con mayoría absoluta en el Parlamento, la promesa de renegociar los términos de la relación del Reino con la UE, para luego convocar a un referéndum que ratificara su eventual acuerdo.

Ahora bien, la pregunta que emerge frente al desarrollo de este fenómeno es por qué se produjo una división tan marcada dentro de los conservadores respecto a la UE, que forzó a Cameron, favorable a la permanencia, a diseñar esta estrategia como modo de saldar el asunto. El argumento de este trabajo es que al menos uno de los motivos centrales viene dado por la lógica de la representación de intereses. En concreto, se plantea la hipótesis de que los parlamentarios que presionaron a los líderes del partido para celebrar un referéndum lo hicieron porque los distritos que representaban demandaban con mayor intensidad salir de la UE que los distritos que eran representados por los legisladores del partido favorables a la permanencia. En Reino Unido, los escaños son asignados en circunscripciones o distritos uninominales, por lo que los parlamentarios electos sienten en ocasiones la presión de actuar como delegados de su circunscripción. Por lo tanto, con el objetivo de aumentar sus posibilidades de reelección $\mathrm{y}$, amenazados por el creciente apoyo obtenido por el Partido por la Independencia del Reino Unido (UKIP, por sus siglas 
en inglés), ${ }^{2}$ la lógica planteada es que estos legisladores se vieron obligados a actuar en su rol de delegados, lo que hicieron mediante el ejercicio de presión al interior de su partido para conseguir la celebración de un referéndum a nivel nacional.

$\mathrm{Si}$ este argumento es correcto, entonces los distritos representados por los legisladores conservadores que apoyaban la opción de la salida deben haber presentado un nivel de votación mayor por esta opción en el referéndum que los distritos representados por los legisladores que apoyaron la permanencia. Por supuesto, este test pone a prueba el argumento solo indirectamente, dado que algunos legisladores pueden haberse posicionado públicamente para el referéndum, pero no haber demandado su celebración al interior del partido anteriormente. De todos modos, dado que el voto por UKIP en las elecciones para el Parlamento Europeo de 2014 está altamente correlacionado con el voto por leave -0.73(Goodwin y Heath, 2016a), es razonable suponer que los legisladores de estas circunscripciones estuvieran enfrentando la presión de representar el deseo de abandonar la UE desde hace un tiempo.

Para testear el argumento se construyó una base de datos con el distrito como unidad de análisis y tres variables: el resultado del referéndum en el distrito, la afiliación partidaria del representante parlamentario del distrito y la posición del representante sobre el referéndum. La información sobre la primera variable viene dada por las estimaciones realizadas por el profesor Chris Hanretty de la Universidad de East Anglia, dado que el referéndum no fue organización por distrito sino por autoridad local, por lo que los resultados oficiales se expusieron a este nivel. ${ }^{3}$ Mientras que la afiliación partidaria y la posición sobre el referéndum surgen de la compilación realizada por el periódico Daily Mirror, que sistematizó esta información para 646 de los 650 integrantes de la Cámara de los Comunes. ${ }^{4}$

\footnotetext{
${ }^{2}$ UKIP, fundado en 1993, es un partido "monotemático", es decir una organización política que tiene como razón de ser la búsqueda de un solo objetivo, en este caso conseguir la salida del Reino Unido de la UE. UKIP había cosechado alrededor del 16\% de los votos en las elecciones europeas de 2004 y 2009, y en las de 2014, con casi el 27\% de los votos, alcanzó el primer lugar, desplazando a los conservadores al tercer puesto (23\%). Por lo tanto, se defiende que la lógica planteada es plausible.

3 Sus estimaciones se encuentran accesibles https://docs.google.com/spreadsheets/d/1wTK5dV2_YjCMsUYlwg0148uWWf44sKgG8uFVMv5OWlA/ed it\#gig=893960794.

${ }^{4}$ La compilación se realizó hasta dos días antes del referéndum, tomando como fuentes de información a cartas, comunicados, tweets, prensa local, campañas, debates y, en ausencia de evidencia en todos estos medios, llamadas a los propios parlamentarios (Daily Mirror 2016). La información se encuentra disponible en http://www.mirror.co.uk/news/uk-news/how-mp-vote-eu-referendum-9187679.
} 
Tras esto, se construyeron tres categorías para clasificar a los distritos. Si el apoyo al leave entre los votantes fue mayor al $55 \%$, se considera que el distrito tiene una fuerte preferencia por la opción de la salida. Por el contrario, si el apoyo fue menor al $45 \%$, se considera que tiene una fuerte preferencia por la permanencia. Para ser conservador, distritos en los que el apoyo al leave se ubicó entre el 45\% y el 55\% se consideraron como "indiferentes" frente a la cuestión de la relación con la UE, dado que es razonable pensar que algunos votantes se decidieron por una de las dos opciones sobre la mesa durante la campaña, y puesto que el resultado por distrito, como se ha explicado, es una estimación. Por todo esto, sería riesgoso asumir que legisladores de estos distritos enfrentaban un claro mandato para presionar por el referéndum al interior del Partido Conservador.

Tabla 1

Posición de los legisladores conservadores en el referéndum por voto por leave en sus distritos

\begin{tabular}{l|ccccc}
\hline \multicolumn{5}{c}{ Voto por leave en distrito } \\
\hline & $<45 \%$ & $45 \%-55 \%$ & $>55 \%$ \\
Posición & Leave & $(13)$ & $31.1 \%(42)$ & $(80)$ & $(135)$ \\
legislador & & $12.9 \%$ & & $42.5 \%$ & $100 \%$ \\
conservador & Remain & $(24)$ & $44.6 \%(83)$ & $(79)$ & $(183)$ \\
\hline
\end{tabular}

Fuente: elaboración propia basada en las estimaciones del resultado por distrito realizadas por Chris Hanretty y en la compilación del Daily Mirror sobre la posición previa de los legisladores frente al referéndum.

Los datos de la tabla 1 muestran que una clara mayoría de los parlamentarios conservadores que se posicionaron en contra de la UE representaban distritos con fuertes preferencias por la salida (59.3\%). El 31,1\% representaba distritos "indiferentes", y menos del 10\% distritos que apoyaban claramente la permanencia. Por el contrario, la mayoría de los parlamentarios del mismo partido que se decantaban por la permanencia representaban distritos "indiferentes" (44,6\%), mientras que $42,5 \%$ representaba distritos en favor del leave y 12,9\% distritos en favor del remain. Adicionalmente, la tabla 2 confirma que el voto promedio por leave en los distritos de legisladores que respaldaban esta opción es más alto que en los distritos de sus colegas que apoyaban al remain (55,9\% vs 52,3\%). Para chequear la relevancia estadística de esta diferencia, se condujo una prueba $T$, la que rechaza que no es significativa a un nivel de confianza del 99\% (ver información adicional en 
el apéndice). Por tanto, la evidencia empírica respalda la lógica de la hipótesis propuesta, esto es, que la división al interior del Partido Conservador sobre la salida de la UE puede explicarse, al menos en parte, por la necesidad de los parlamentarios del partido de representar distritos con preferencias divergentes sobre este tema.

Tabla 2

Comparación de medias de voto por leave en distritos representados por legisladores conservadores discriminados por posición frente al referéndum

\begin{tabular}{lcccc}
\hline $\begin{array}{l}\text { Posición } \\
\text { legislador } \\
\text { conservador }\end{array}$ & $\mathrm{N}$ & Media & Desv. Estándar & $\begin{array}{c}\text { Media de error } \\
\text { estándar }\end{array}$ \\
\hline Remain & 186 & $53.3 \%$ & $9 \%$ & $1 \%$ \\
\hline Leave & 135 & $55.9 \%$ & $8 \%$ & $1 \%$ \\
\hline
\end{tabular}

Fuente: elaboración propia basada en las estimaciones del resultado por distrito realizadas por Chris Hanretty y en la compilación del Daily Mirror sobre la posición previa de los legisladores frente al referéndum.

Ahora bien, una vez que existe evidencia que respalda una lógica de representación de intereses, aparece la interrogante de cuáles son dichos intereses. Como se ha visto, la literatura predominante, sintetizada en el enfoque OEP, considera a los intereses como ganancias en términos materiales, y asume que las preferencias de individuos y grupos sobre distintas opciones de política económica pueden ser derivadas de su posición en la estructura de la economía, pues estas permiten identificar el impacto esperado de las alternativas en disputa (Lake, 2009). Sin embargo, la utilización de esta estrategia en el caso del Brexit es, por lo menos, problemática. Esto se debe a que ni la modalidad de la salida ni los términos de la futura relación económica con la UE estaban determinados de antemano, y las consecuencias distributivas de las eventuales alternativas difieren considerablemente. Además, por la existencia de un mercado común dicha relación involucra múltiples áreas (comercial, financiera, de inversión, migratoria), por lo que identificar a los eventuales ganadores y perdedores es un ejercicio nada evidente.

Ante esta situación de indefinición, una alternativa es identificar características que discrimen entre aquellos que votaron por la salida y aquellos que lo hicieron por la permanencia. Distintos estudios socio-demográficos han encontrado que las personas con bajos niveles de educación formal, edad avanzada, trabajadores manuales con salarios estancados y residentes de regiones afectadas por la desindustrialización, la mayor competencia de importaciones provenientes de los 
nuevos centros industriales como China y una creciente desigualdad, fueron los principales partidarios del leave (Becker et al., 2016; Colantone y Stanig, 2018; Darvas, 2016; Financial Times, 2016; Goodwin y Heath, 2016a; 2016b; Langella y Manning, 2016; The Economist, 2016a). Estos trabajos concluyen que este voto puede ser entendido como una respuesta de aquellos "dejados atrás" por la globalización o, en otras palabras, de individuos que atraviesan situaciones relativamente prolongadas de inseguridad económica producto de una mayor competencia económica a nivel mundial. En algunas explicaciones, incluso el contexto geográfico sería un determinante más importante que la situación individual, es decir que los votantes se comportarían de una forma "sociotrópica", respondiendo políticamente a la situación económica de su entorno. Si este fuera el caso, en cualquiera de estas dos variantes explicativas, entonces uno podría inferir que los legisladores promotores del Brexit estaban representando los intereses materiales de los perdedores de la globalización.

Sin embargo, esta interpretación choca con el hecho de que la campaña a favor del leave no fue principalmente presentada como una herramienta de compensación hacia los individuos y las regiones afectadas por los cambios en la economía global; de hecho, los votantes con tendencia a manifestar preocupación por el impacto de un Brexit en la economía y el empleo apoyaron mayoritariamente la permanencia. Por el contrario, los votantes del leave manifestaban mayoritariamente entre sus preocupaciones la inmigración y la pérdida de soberanía nacional frente a la UE (Lord Ashcroft, 2016; Prosser et al, 2016).

Una explicación alternativa explora el rol de los valores sociales conservadores y la "política de la identidad”. En esta línea, estudios han reportado asociaciones entre el apoyo hacia el leave y el rechazo del multiculturalismo y otros valores liberales, auto-identificación como "inglés" en vez de "británico" e incluso apoyo por la pena de muerte (Lord Ashcroft, 2016; Kaufmann, 2016). En esta mirada, el voto a favor del Brexit es entendido como una reacción contra los cambios en los valores y tradiciones más que la consecuencia de pérdidas materiales. Sin embargo, este argumento olvida que tanto históricamente (Polanyi, 1944), como actualmente (Burgoon, 2009), el ascenso del nacionalismo y otras expresiones intensas de identidad están asociados con el incremento del flujo transnacional de bienes y factores de producción, inseguridad económica y ausencia de mecanismos efectivos de compensación o redes de protección. Este parece ser el caso del Reino Unido, un país bien integrado en la economía mundial pero en el que influyentes expertos como Mark Carney, gobernador del Banco de Inglaterra (banco central), o el economista jefe de la misma institución, Andrew Haldane, pasando por revistas especializadas como The Economist, advierten sobre una creciente brecha entre 
regiones en términos de ingresos y productividad, con la consecuente mayor desigualdad que no obstante no es compensada con políticas activas que la moderen (BBC, 2016a; 2016b, The Economist, 2016a).

Esta idea de que los fenómenos de deterioro económico relativo y reacciones identitarias se encuentran relacionadas ha sido testeada recientemente por abordajes cuantitativos. Por ejemplo, Colantone y Stanig (2018) muestran que la mayor competencia comercial en las regiones tradicionalmente manufactureras del Reino Unido, combinadas con la ausencia o la retracción de políticas de compensación hacia los perdedores (en el marco de los programas de austeridad implementados tras la crisis global del 2008), dan cuenta a la vez de: 1) una mayor propensión individual al rechazo a la inmigración y 2) una mayor proporción de apoyo al leave (Colantone y Stanig, 2018). Y en la misma línea, evidencia anecdótica y revelada por encuestas coincide en señalar un vínculo entre reacciones identitarias y quejas "económicas" sobre los supuestos efectos negativos de la inmigración en los salarios y en las presiones sobre el Estado de Bienestar (The Economist, 2016b, YouGov, 2016). Por ende, para entender como esta retroalimentación entre intereses materiales y reacciones identitarias ha influenciado el Brexit, puede ser de ayuda atender al rol de las ideas y las narrativas.

Para esto, es preciso retomar la literatura que, a contrapelo del enfoque OEP, sostiene que ni la transformación de una situación material determinada en “intereses", ni la posterior transformación de intereses en preferencias sobre política pública, es automática (Rodrik, 2014). Y, a su vez, que dicha transformación tampoco ocurre a nivel individual, sino que se da en el marco de interacciones sociales entre distintos actores que determinan una construcción intersubjetiva en un momento dado del tiempo (Woll, 2008). Así, esta línea de pensamiento recurre a las visiones sobre el mundo, los supuestos predominantes y otros dispositivos ideacionales para entender cómo los grupos y los individuos que los componen definen sus intereses y escogen caminos para satisfacerlos.

Siguiendo esta lógica de razonamiento, puede ser argumentado que luego de una crisis financiera como la vivida en 2008, situación en que las ideas parecen ser particularmente poderosas (Blyth, 2002), dos narrativas sobre las fuentes de los problemas del Reino Unido entraron en competencia. Una culpa al sector financiero y a las políticas de austeridad que siguieron a la crisis, mientras que la otra se enfoca en los efectos de la inmigración y la pérdida de soberanía debido al "avance" de Bruselas. Una encuesta de YouGov de 2016 ilustra esta diferencia: para los partidarios de la permanencia, las tres principales causas de los problemas británicos eran los bancos, el gobierno conservador y la creciente desigualdad; para los partidarios de la salida, los equivalentes eran las regulaciones excesivas de la UE, 
los inmigrantes dispuestos a trabajar por bajos salarios y el anterior gobierno laborista (YouGov, 2016). Evidentemente, la segunda narrativa ha sido más exitosa. Los motivos de esto sin dudas merecen mayor investigación, aunque el rol de UKIP como un emprendedor político capaz de desplegar este mensaje entre los perdedores de los fenómenos económicos recientes es un punto de partida necesario. En todo caso, lo importante a resaltar aquí es que mediante una conexión discursiva entre la UE y los problemas de los británicos, se logró construir exitosamente un "interés” en el Brexit entre los votantes desfavorecidos por su situación económica y la de sus regiones.

El éxito de esta narrativa también contribuye a entender por qué todos menos 10 parlamentarios laboristas apoyaron la permanencia, a pesar de que 70\% de ellos representan distritos que votaron mayoritariamente por la salida (Hanretty, 2016). ${ }^{5}$ La manera en que el Brexit fue presentado por sus promotores no era aceptable para ninguna de las alas del laborismo, incluso para su sector más euroescéptico, representado entre otros por su líder actual Jeremy Corbyn. Para Corbyn y sus aliados políticos, la UE puede suponer una barrera indeseable frente a la alternativa de un mayor rol del Estado en términos de política industrial (por ejemplo, por sus reglas de ayuda estatal), o limitar la expansión de las políticas de bienestar producto de sus restrictivas reglas fiscales. Sin embargo, la alternativa de salirse para limitar la inmigración, o bien para promover una mayor desregulación de las actividades económicas, motivos de los promotores del Brexit, generan en este sector incluso más rechazo. Así, como señaló con algo de resignación el parlamentario laborista Stephen Kinnock, en una conferencia dictada en la London School of Economics, en este tema era en lo único, aunque claro que por distintas razones, en lo que las actuales y enfrentadas alas de su partido podían coincidir. Para los seguidores de la vía centrista impulsada por el ex primer ministro Tony Blair, el Brexit era un atentado contra la globalización que entusiásticamente apoyan; para los seguidores de la vía izquierdista de Corbyn, aunque la UE sea ante sus ojos un proyecto neoliberal, el discurso anti-inmigración que lo promueve no es más que una expresión de racismo (Kinnock, 2016).

Por el contrario, los conservadores podían adaptarse más cómodamente a un discurso que subraya la necesidad de preservar las tradiciones británicas, incluso si esto implicaba restringir la inmigración y defender la soberanía parlamentaria de Westminster frente a la UE. Por ende, un grupo de ellos siguió dicho camino como estrategia para mantener e incluso mejorar sus perspectivas electorales. Si este argumento es correcto, entonces el Brexit puede ser entendido como una peculiar

\footnotetext{
${ }^{5}$ Lo que es más, los 10 “disidentes” representan distritos favorables a la permanencia.
} 
combinación de dinámicas de representación y de construcción de intereses, que ocurren en un nuevo marco de conductas políticas producidas como reacción a los costos impuestos por la globalización económica en determinados individuos y regiones de los países avanzados. Estas conductas abren oportunidades y desafíos para los políticos que participan en la competencia electoral. Pues, por un lado, pueden procurar influenciarlas mediante su rol de emprendedores; pero, una vez consolidadas, como en el caso del Brexit, también se enfrentan a la necesidad de ajustar su accionar para representarlas o quedar relegados electoralmente.

\section{Impactos sobre las teorías del regionalismo}

El Brexit es sin dudas un fenómeno de dimensiones significativas. En términos absolutos, el Reino Unido es la quinta economía más grande del planeta y la segunda en importancia de toda la UE, tan solo por detrás de Alemania (FMI, 2016). Mientras que la propia UE es el bloque económico de mayor tamaño del globo, y representa a la vez el proceso de integración regional más profundo, en tanto la coordinación intergubernamental es complementada con la presencia de instituciones de carácter supranacional. Por lo tanto, la decisión de los británicos genera un impacto tanto a nivel de las teorías sobre el regionalismo como sobre el propio proceso europeo. Pero, además, el Brexit se inscribe dentro de un conjunto de políticas de desintegración recientes, algunas esbozadas aunque no concretadas, como la amenaza de la administración Trump en Estados Unidos de retirarse del Tratado de Libre Comercio de América del Norte (TLCAN), o las recientes declaraciones de Jair Bolsonaro, presidente de Brasil, sobre una eventual salida de su país del Mercosur. Así, la consideración del Brexit ofrece una oportunidad para repensar las teorías que buscan explicar la conformación de acuerdos de integración económica regional.

La emergencia de iniciativas de integración regional a principios de la década de 1990 renovó el debate académico sobre los factores que propician el regionalismo, que anteriormente se había centrado de forma casi exclusiva en las discusiones entre federalistas, neofuncionalistas e intergubernamentalistas sobre el proceso de integración en Europa. En un nuevo escenario internacional signado por el fin de la guerra fría, algunos enfoques vincularon la emergencia de espacios regionales con las dinámicas de la política del comercio que tenían lugar a nivel multilateral. Así, por ejemplo, Mansfield y Reinhardt (2003), sostuvieron que la creciente membresía de la Organización Mundial del Comercio (OMC), la presencia de rondas de negociación multilateral y la pérdida de disputas comerciales en los paneles de 
solución de controversias en la OMC eran los principales determinantes del regionalismo.

Dado que el otorgamiento de preferencias regionales introduce una discriminación sobre las condiciones de acceso a mercados acordadas a nivel multilateral, las nuevas teorías del regionalismo proyectaron que estos procesos tendrían un crecimiento endógeno, es decir, motivado por su propia existencia. En este sentido destaca el trabajo de Baldwin (1993; 1997), quien propuso una teoría del dominó del regionalismo. De acuerdo a Baldwin, una vez que un grupo de países decide otorgarse mutuamente preferencias comerciales mejores a las establecidas por la cláusula de la nación más favorecida, los exportadores de terceros países tienen incentivos adicionales para presionar a sus gobiernos para unirse a estos acuerdos, pues de otra forma su capacidad competitiva se ve afectada de forma negativa. Esta movilización cambia el equilibrio político entre los promotores de la liberalización del comercio y los promotores de protección a favor de los primeros, lo que resulta en nuevas membresías. Y a medida que las organizaciones regionales se extienden, los incentivos para el ingreso se difunden hacia terceros países.

Baccini y Dür (2011) plantean una dinámica similar, en la que la competencia por mercados es el motor del nuevo regionalismo. Su argumento, que construye sobre los aportes de Baldwin, se basa en que el desvío de comercio moviliza a los exportadores de terceros mercados, llevando a sus gobiernos a la necesidad de "protegerlos" por medio de la conformación de nuevos acuerdos comerciales. Esta dinámica también ha sido identificada en la formación de acuerdos en las Américas, en particular para explicar la conclusión de tratados de comercio "Norte-Sur", esto es, entre países desarrollados y países en desarrollo. El argumento es que "el miedo a ser excluido" por la formación de acuerdos entre competidores regionales y los principales mercados de exportación (especialmente Estados Unidos), lleva a los países a una carrera por asegurar sus propios acuerdos con el hub (SánchezAncochea, 2008; Shadlen, 2008; Quiliconi, 2014). Como Gruber (2001) explica, países como Estados Unidos tienen la capacidad, mediante iniciativas unilaterales, de remover el statu quo como opción, llevando a terceros países a tener que modificar sus políticas. De acuerdo a este autor, una lógica similar se observa en la propia UE, lo que explicaría la adhesión tardía de países inicialmente reticentes, producto de sus preferencias divergentes del resto en términos del nivel deseado de igualdad social, como Austria, Finlandia y Suecia.

Como se desprende de su repaso, todos estos enfoques se centran en las tendencias hacia la absorción de nuevos miembros una vez que las economías con mayor capacidad de atracción cambian su rumbo de estrategia comercial. Las presiones del ambiente, señalan, dejan sin margen de alternativa a los gobiernos a la hora de 
decidir. En otras palabras, su argumento central es que existe un camino unidireccional al progresivo crecimiento del regionalismo. Sin embargo, el Brexit mostró los límites de dicho entendimiento. La decisión del Reino Unido de salir de la UE pone al descubierto que a pesar de las presiones enfatizadas por la literatura sobre el regionalismo, que efectivamente existen, también operan fuerzas en sentido contrario que los modelos existentes no logran captar. Bajo qué condiciones pueden desatarse, y cuáles son los factores que las impulsan, son aspectos sobre los que aún existe un grado muy bajo de entendimiento.

Un primer elemento a tener en consideración en cuanto a condiciones es el tamaño de la economía británica. Esto es, la capacidad económica del país le brinda un conjunto de oportunidades que habilitan decisiones tal vez impensadas para otros Estados. Tan solo como ejemplo, durante la crisis de la deuda en Grecia se manejó la posibilidad de una salida de la Unión. No obstante, los sucesivos gobiernos griegos, aun siendo de distinto signo político, hicieron todo lo posible por la permanencia, incluso aunque esto supusiera ejecutar acciones tales como desconocer el resultado de un referéndum y aplicar un conjunto de medidas con enormes consecuencias sociales en el corto plazo. De esto se desprende que tal vez las nuevas teorías del regionalismo han prestado atención a las disyuntivas enfrentadas por países pequeños o medianos, pero que como el Brexit releva no necesariamente aplican a economías de mayor porte.

Por otra parte, en cuanto a los factores que impulsan las decisiones de desintegración, la sección precedente mostró que no necesariamente se asocian a un deterioro económico causado de forma directa por la membresía, pues no existen elementos para trazar una conexión entre la participación (salida) del Reino Unido en la UE y un deterioro (mejoramiento) en las condiciones económicas de los británicos. Sin embargo, sí se registró la importancia de una caída relativa de las condiciones de vida de segmentos importantes de la población, producto de otros fenómenos como la mayor competencia de los centros de producción asiáticos en el marco de la globalización, y la retracción de las políticas de bienestar tras la crisis financiera de 2008. Y a esto se agregó la importancia de las ideas y narrativas impulsadas por emprendedores políticos, que modelaron las preferencias ciudadanas hacia orientaciones contrarias a la inmigración y a la pérdida de la soberanía parlamentaria como reacción. Cierto es que poco sabemos aún sobre cómo se construyen estas narrativas, quiénes son capaces de construirlas y quiénes ganan y pierden con ellas, lo que abre nuevas vías para la investigación. Pero lo cierto es que en la actualidad, globalización y regionalismos conviven con nuevos elementos que tienden hacia la fragmentación. 
Asimismo, es interesante notar los paralelismos que emergen entre el Brexit y la elección de Trump en Estados Unidos. Pues las mismas variables, tales como una mayor competencia de las exportaciones provenientes de Asia (en particular China) sobre los principales centros manufactureros del país, sumado a un estancamiento de los ingresos reales de los trabajadores industriales, profundizado tras la crisis de 2008, explican de acuerdo a algunos estudios (ej. Autor et al., 2017; Jensen et al., 2017) el apoyo de votantes tradicionalmente demócratas a la plataforma electoral proteccionista y antiinmigración (en ocasiones con ribetes xenófobos) del candidato republicano. ${ }^{6}$

Un abordaje con potencial para entender este nuevo tipo de fenómenos es el trilema político de la economía mundial, planteado hace ya unos años por Rodrik (2011). Este autor sostiene que hiperglobalización, soberanía estatal y política democrática son incompatibles, pues en el mejor de los casos se puede contar con dos, pero no con los tres atributos mencionados, a la vez. Esto lleva a la elaboración de tres escenarios, descritos en la figura 1, que reflejan todas las combinaciones de pares posibles: una camisa de fuerza dorada en donde se mantiene la hiperglobalización y la soberanía nacional, a la expensa de la política democrática; una gobernanza global o regional en donde hiperglobalización y decisiones democráticas ocurren a un nivel superior al del Estado nacional, por lo que se resigna la soberanía nacional; y un tercer escenario llamado compromiso de Bretton Woods en donde se limita la hiperglobalización para mantener la política democrática a nivel del Estado nacional.

La UE fue clasificada por Rodrik, en su libro de 2011, como un ejemplo de gobernanza a un nivel superior del Estado nacional, en donde la política democrática comenzaba a tener lugar progresivamente a nivel regional a costa de la pérdida de ciertos márgenes de soberanía estatal. Sin embargo, evaluaciones más recientes del propio Rodrik (2016) así como de otros académicos cuestionan la efectividad de los mecanismos de democracia a nivel europeo, señalando la existencia de problemas de representación tanto por la lejanía de las instituciones representativas como por el rol sustantivo de técnicos no electos en la toma de decisiones políticas. Así, el Brexit aparecería como una respuesta desde la política por "tomar nuevamente el control", tal como plantean sus proponentes, lo que significaría recuperar espacios para la política democrática a nivel del Estado nacional.

\footnotetext{
${ }^{6}$ Agradezco a un revisor anónimo por sugerirme abordar este paralelismo.
} 
Figura 1

El trilema político de Rodrik

\section{El trilema político de la economía mundial}

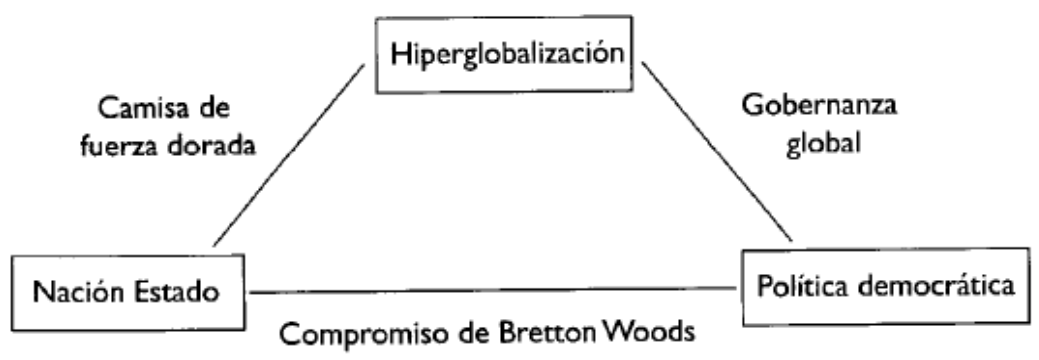

Figura 9-1. Elija dos.

Fuente: Rodrik (2011: 219)

Sin embargo, al mismo tiempo, las principales figuras del leave plantean como alternativa a la UE la promoción de un Reino Unido de alcance "global" -o lo que es lo mismo, híperglobalizado-, con acuerdos comerciales que incluyen varias disciplinas relacionadas con el comercio que limitan la formulación de alternativas a nivel doméstico. Así, el Brexit sería un ejercicio puntual de política democrática que busca reencauzar al país hacia el escenario de camisa de fuerza dorada, recuperando eso sí la soberanía para formular este tipo de decisiones. ${ }^{7}$ Por supuesto, esta propuesta política compite con, por ejemplo, aquella formulada por el laborismo actual, en donde la vuelta hacia un esquema similar al compromiso de Bretton Woods parece ser la opción preferida. Pero más allá de estas apreciaciones coyunturales, lo cierto es que los fenómenos observados nos llevan a la necesidad de ampliar el espectro teórico posible de respuesta tras la irrupción de la política en la reconfiguración de las estrategias de relacionamiento con la economía mundial de algunos de sus principales jugadores.

De todos modos, también debe destacarse que las tendencias hacia la fragmentación conviven con impulsos hacia el regionalismo, y que en ocasiones incluso ambos fenómenos se encuentran interrelacionados. Esto se observa claramente en el caso de la UE. La decisión de los británicos, sumada a la crisis de los refugiados y a la pobre recuperación económica de la eurozona tras el colapso financiero y económico de 2008, parecía configurar una tormenta perfecta que amenazaba el

\footnotetext{
${ }^{7}$ Igualmente, se podrían realizar consideraciones sobre los potenciales ganadores y perdedores de la agenda económica del caso ya referido de la administración Trump en Estados Unidos, lo que no obstante escapa al alcance de este trabajo.
} 
proceso regional más consolidado del mundo. En el este, las llamadas "democracias iliberales" de Hungría y Polonia introducían elementos que cuestionan la separación de poderes y el estado de derecho, y se oponían vehementemente a todo intento de la Comisión Europea de establecer cuotas obligatorias para el establecimiento de refugiados. Controles en las fronteras, cuyo removimiento simboliza la profundidad del regionalismo europeo, comenzaron a ser reintroducidos. En el sur, el sistema bancario italiano no mostraba ningún signo de recuperación, y distintos movimientos políticos de importancia en el sistema político italiano, como la Liga y el Movimiento 5 Estrellas, comenzaron a manejar la idea de un "Italexit", en el marco de su novel acceso al gobierno en coalición. En el norte, gobiernos e importantes segmentos del electorado en países como Alemania, pero también Finlandia y Holanda, mostraban su creciente reticencia a financiar lo que veían como un comportamiento económicamente irresponsable de sus vecinos del sur. $\mathrm{Y}$ en Francia, núcleo central del regionalismo europeo en conjunto con Alemania desde la segunda posguerra, una candidata con un discurso abiertamente eurófobo encabezaba en los sondeos de opinión de cara a las elecciones presidenciales del país en 2017.

Sin embargo el Brexit, junto a los otros elementos mencionados, actuaron como catalizadores de medidas necesarias pero que encontraban fuerte resistencia en el espacio europeo. En este sentido, en particular, se destaca el apoyo al programa de aflojamiento cuantitativo llevado adelante por el Banco Central Europeo desde inicios de 2015. Inicialmente, dicho programa encontró amplias resistencias en Alemania y otros países del norte de Europa, cuyas economías no necesitaban de tasas de interés cercanas al 0\%, al estilo de las aplicadas en Estados Unidos o el Reino Unido. No obstante, la combinación de políticas monetaria y fiscal restrictivas aplicadas hasta ese momento obligaba a los países de la periferia de la eurozona a ensayar devaluaciones internas con enormes consecuencias sociales en términos de empleo y bienestar (De Grauwe 2013; Krugman 2012). Estos efectos alimentaban la creciente insatisfacción de los ciudadanos frente a las instituciones europeas, abriendo nuevos flancos de batalla. Pero en tanto el Brexit y otros desafíos amenazaban con mayor intensidad el proyecto europeo, los socios del norte comenzaron a aceptar medidas con las que previamente mostraban poca o ninguna tolerancia.

La evidencia más reciente que emerge desde Europa confirma esta tendencia. En junio de 2017, Italia rescató dos de sus bancos regionales por un valor de 1700 millones de euros (Financial Times, 2017). Este tipo de salvatajes está prohibido bajo las reglas que gobiernan la UE, pero de todos modos las autoridades italianas 
encontraron algunas grietas legales para llevarlos adelante. Sin embargo, el punto más importante para este análisis es que Alemania y sus socios nórdicos no se movilizaron para evitarlos, lo que refleja la nueva predisposición a aceptar una mayor flexibilidad con las economías que arrastran mayores problemas.

Esto no significa que la UE no continúe enfrentando desafíos de gran porte: las diferencias estructurales entre los socios continúan, y las soluciones ensayadas hasta el momento, como el aflojamiento cuantitativo, tienen una naturaleza temporal. Además, las identidades y tradiciones nacionales permanecen en tensión con la visión de las instituciones supranacionales, como los enfrentamientos recientes entre la Comisión Europea y Polonia reflejan. ${ }^{8}$ Y la introducción de alguna palanca fiscal regional, necesaria para equilibrar los desfasajes en los ciclos económicos de los miembros de la Eurozona, no termina de concretarse. De todos modos, y un tanto irónicamente, es posible concluir que el Brexit ha contribuido a reducir y no a exacerbar estos desafíos, al menos en el corto plazo.

\section{Conclusiones}

Fenómenos complejos como el Brexit, que suponen rupturas sistémicas con amplias repercusiones en términos de reconfiguraciones regionales y globales, ponen a prueba la capacidad de los enfoques existentes de dar cuenta de la realidad. La salida de la UE de la quinta economía mundial y segunda del bloque regional demanda esfuerzos adicionales para entender tanto sus causas como sus consecuencias. Este trabajo intentó mostrar, en primer lugar, que a pesar de que la decisión tomada por los británicos fue el resultado directo de un referéndum, parte de la explicación del Brexit viene dada por la lógica de la representación de intereses. Pues las divisiones registradas al interior del Partido Conservador, que llevaron a la convocatoria de esta consulta popular, encuentran su razón en la necesidad de los parlamentarios tories, que compiten en distritos uninominales, de representar intereses divergentes de sus electores respecto a la relación deseada con la UE.

De todos modos, también se mostró que estos intereses no se entienden mediante la aplicación de las teorías estándares sintetizadas en el enfoque OEP, en tanto el Brexit no fue planteado desde la lógica de compensar a los perdedores de la globalización, quienes supusieron el grueso del respaldo electoral por esta opción, sino desde un discurso reivindicativo de la identidad inglesa, sospechoso de la

\footnotetext{
${ }^{8}$ Dicho conflicto gira en torno a la independencia política del Poder Judicial en el país, la que de acuerdo a la UE se encuentra comprometida con las reformas introducidas desde 2016, tras la llegada al gobierno del partido Ley y Justicia. Actualmente, la Comisión Europea mantiene abierto un procedimiento contra Polonia por dicha cuestión.
} 
transferencia de potestades regulatorias a Bruselas y opuesto a la inmigración. Por tanto, el trabajo sugiere atender al rol de las ideas y las narrativas en la construcción de intereses y preferencias de política pública, aspecto que demanda una mayor investigación futura.

Por otra parte, se destaca que la decisión de los británicos expone los límites de las teorías del regionalismo que predicen un camino unidireccional hacia la conformación de acuerdos regionales, en donde nuevos miembros se unen a esta tendencia y ninguno muestra políticas en la dirección opuesta. Como se ha dicho, el Brexit es hasta el momento el caso más ilustrativo, pero la opción manejada por Trump de retirarse del TLCAN en el marco de su renegociación, su retiro del Trans Pacific Partnership antes de su entrada en vigor, o incluso las declaraciones de Bolsonaro respecto a la permanencia de Brasil en el Mercosur, revelan que es parte de una tendencia más amplia. Estos fenómenos sugieren la necesidad de nuevas teorías de que den cuenta de la evidencia mixta hacia la integración y la desintegración que actualmente se observa en la economía mundial. En este sentido, el tamaño de la economía de quienes se proponen ejercer opciones de salida aparece como relevante. Pero, además, estas decisiones se dan en el marco del trilema político planteado por Rodrik (2011), en donde la demanda por un mayor control democrático de la toma de decisiones, especialmente en contextos donde las políticas producen o no compensan a los perdedores, aparece como factor determinante. Dicho esto, las alternativas que se formulan apuntan en distintas direcciones, no siempre consistentes con las demandas previas, lo que abre todo un complejo campo de investigación para futuros estudios.

Finalmente, es preciso mencionar que la evidencia emergente tampoco sugiere que deba esperarse una cascada en sentido contrario al registrado en las últimas décadas, esto es, un proceso de fragmentación unidireccional. Pues de hecho, como se ha argumentado, la UE ha tendido a reforzarse, más que a debilitarse, tras la decisión de los británicos. En conclusión, en Europa y, posiblemente en otras regiones, conviven fuerzas que tienden a la fragmentación con otras que refuerzan los procesos de integración existentes, con el agregado de que se encuentran interrelacionadas.

\section{Referencias bibliográficas}

Autor, D. et al. (2017). Importing Political Polarization? The Electoral Consequences of Rising Trade Exposure. NBER Working Paper 22637. 
Baccini, L. y Dür, A. (2012). The New Regionalism and Policy Interdependence. British Journal of Political Science, 42 (1), 57-79.

Baldwin, R. (1993). A domino theory of regionalism. NBER Working Paper 4465.

Baldwin, R. (1997) The Causes of Regionalism. The World Economy, 20(7), 865-888.

BBC (2011). EU referendum. Rebels lose vote Commons. En linea http://www.bbc.co.uk/news/uk-politics-15425256. Último acceso 20/8/2019.

BBC (2016a). BoE's Andrew Haldane warns of regional growth inequality. En línea http://www.bbc.co.uk/news/business-38186053. Último acceso 20/8/2019.

BBC (2016b). Carney warns about popular disillusion with capitalism. En línea http://www.bbc.co.uk/news/business-38210169. Último acceso 20/8/2019.

Becker, S. et al. (2016). Who Voted for Brexit? A Comprehensive District-Level Analysis. CEPR Discussion Paper No. 11954.

Blyth, M. (2002). Great Transformations: Economic Ideas and Institutional Change in the Twentieth Century. Nueva York: Cambridge University Press.

Burgoon, B. (2009). Globalization and backlash: Polayni's revenge? Review of International Political Economy, 16(2), 145-177.

Business-Insider UK (2016). This is the size of the majority in the House of Commons against Brexit. En línea http://uk.businessinsider.com/majority-house-ofcommons-against-brexit-2016-11. Último acceso 20/8/2019.

Colantone, I. y Stanig, P. (2018). Global Competition and Brexit. American Political Science Review, 112(2), 201-218.

Daily Mirror (2016). How did my MP vote in the Brexit referendum? Search by name or constituency after Brexit ruling. En línea http://www.mirror.co.uk/news/uknews/how-mp-vote-eu-referendum-9187679. Último acceso 20/8/2019.

Darvas, Z. (2016). Brexit Should Be a Wake Up Call in the Fight against Inequality. LSE European Politics and Policy Blog.

De Grauwe, P. (2013). The Political Economy of the Euro. Annual Review of Political Science, 16(1), 153-170. 
Financial Times (2016). UK areas with wage stagnant are most anti-EU. En línea https://www.ft.com/content/fe5c6b4e-32f8-11e6-bda0-04585c31b153. Último acceso 20/8/2019.

Financial Times (2017). Why Italy's €17bn bank deal is making waves across Europe. En línea https://www.ft.com/content/fe5c6b4e-32f8-11e6-bda0-04585c31b153. Último acceso 20/8/2019.

FMI (2016). World Economic Outlook database.

Goodwin, M. y Heath, O. (2016a). Brexit vote explained: poverty, low skills and lack of opportunities. En linea https://www.jrf.org.uk/report/brexit-vote-explainedpoverty-low-skills-and-lack-opportunities. Último acceso 20/8/2019.

Goodwin, M. y Heath, O. (2016b). The 2016 Referendum, Brexit and the Left Behind: An Aggregate-Level Analysis of the Results. Political Quarterly, 87(3), 323-332.

Gruber, L. (2001). Power Politics and the Free Trade Bandwagon. Comparative Political Studies, 34 (7), 703-741.

Hanretty, C. (2016). Most Labour MPs represent a constituency that voted $R$. En linea https://medium.com/@chrishanretty/most-labour-mps-represent-aconstituency-that-voted-leave-36f13210f5c6\#.x2vkrjcrd. Último acceso 20/8/2019.

Hiscox, M. (2014). The Domestic Sources of Foreign Economic Policies. En Ravenhill, John (ed.) Global Political Economy. Oxford: Oxford University Press, 74105.

Jensen, B. et al. (2017). Winners and Losers in International Trade: The Effects on US Presidential Voting. International Organization, 71(3), 423-457.

Kaufmann, E. (2016). It's NOT the economy, stupid. Brexit as a story of personal values. LSE Blog British Politics and Policy. En línea http://blogs.lse.ac.uk/politicsandpolicy/personal-values-brexit-vote/. Último acceso 20/8/2019.

Kinnock, S. (2016). Intervention in the conference The New Minority: white working class politics in an era of immigration and inequality. 21 de noviembre, London School of Economics, Londres. Documento de audio, en línea http://richmedia.lse.ac.uk/publiclecturesandevents/20161121_1800_theNe wMinority.mp3. Último acceso 20/8/2019. 
Krugman. P. (2012). Revenge of the Optimum Currency Area. The New York Times. En línea http://krugman.blogs.nytimes.com/2012/06/24/revenge-of-theoptimum-currency-area/?_r=0. Último acceso 20/8/2019.

Lake, D. (2009). Open economy politics: A critical review. The Review of International Organizations, 4(3), 219-244.

Langella, M. y Manning, A. (2016). Who Voted Leave?. CentrePiece Autumn. En línea http://cep.lse.ac.uk/pubs/download/cp479.pdf. Último acceso 20/8/2019.

Lord Ashcroft (2016). How the United Kingdom voted on Thursday... and why. En línea http://lordashcroftpolls.com/2016/06/how-the-united-kingdom-voted-andwhy/. Último acceso 20/8/2019.

Mansfield, E. y Reinhardt, E. (2008). Multilateral Determinants of Regionalism: The Effects of GATT/WTO on the Formation of Preferential Trading Arrangements. International Organization, 57(4), 829-862.

Milner, H. y Judkins, B. (2004). Partisanship, Trade Policy and Globalization: Is There a Left-Right Divide on Trade Policy?. International Studies Quarterly, 48 (1), 95119.

Polanyi, K. (1944). The Great Transformation. New York: Farrar \& Rinehart.

Prosser, C. et al. (2016). What mattered most to you when deciding how to vote in the EU referendum?. British Election Study Team. En línea http://www.britishelectionstudy.com/bes-findings/what-mattered-most-toyou-when-deciding-how-to-vote-in-the-eu-referendum/\#.WEQxpubJxPY . Último acceso 20/8/2019.

Quiliconi, C. (2014). Competitive Diffusion of Trade Agreements in Latin America. International Studies Review, 16(2), 189-208.

Rodrik, D. (2011). La paradoja de la globalización. Barcelona: Antoni Bosch.

Rodrik, D. (2014). When Ideas Trump Interests: Preferences, Worldviews, and Policy Innovations. Journal of Economic Perspectives, 28(1), 189-208.

Rodrik, D. (2016). Brexit and the Globalization Trilemma. En línea https://rodrik.typepad.com/dani_rodriks_weblog/2016/06/brexit-and-theglobalization-trilemma.html. Último acceso 20/8/2019. 
Sánchez-Ancochea, D. (2008). State and Society: The Political Economy of DR-CAFTA in Costa Rica, the Dominican Republic, and El Salvador. En Sánchez-Ancochea, Diego y Shadlen, Ken (eds.), The Political Economy of Hemispheric Integration. Responding to Globalization in the Americas. New York: Palgavre Macmillan, 171-200.

Schonhardt-Bailey, C. (2006). From the Corn Laws to Free Trade: Interests, Ideas and Institutions in Historical Perspective. Cambridge: MIT Press.

Shadlen, K. (2008). Globalization, Power, and Integration: The Political Economy of Regional and Bilateral Trade Agreements in the Americas. Journal of Development Studies, 44(1), 1-20.

The Economist (2016a). Collateral damage. The impact of Free Trade. En línea http://www.economist.com/news/britain/21702791-britain-unusually-opentrade-unusually-bad-mitigating-its-impact-collateral-damage. Último acceso 20/8/2019.

The Economist (2016b). Global Politics. League of Nationalists. En línea http://www.economist.com/news/international/21710276-all-aroundworld-nationalists-are-gaining-ground-why-league-nationalists. Último acceso 20/8/2019.

Woll, C. (2008) Firm Interests: How Governments Shape Business Lobbying on Global Trade, Ithaca: Cornell University Press.

YouGov (2016). EU referendum: Provincial England versus London and the Celts. En línea https://yougov.co.uk/news/2016/03/24/eu-referendum-provincialengland-versus-london-and/. Último acceso 20/8/2019.

\section{Apéndice}

\begin{tabular}{|c|c|c|c|c|c|c|c|c|c|c|}
\hline \multicolumn{11}{|c|}{ Independent Samples Test } \\
\hline & & \multicolumn{2}{|c|}{$\begin{array}{c}\text { Levene's Test for Equality of } \\
\text { Variances }\end{array}$} & \multicolumn{7}{|c|}{ t-test for Equality of Means } \\
\hline & & \multirow[b]{2}{*}{$\mathrm{F}$} & \multirow[b]{2}{*}{ Sig. } & \multirow[b]{2}{*}{$t$} & \multirow[b]{2}{*}{ df } & \multirow[b]{2}{*}{ Sig. (2-tailed) } & \multirow{2}{*}{$\begin{array}{l}\text { Mean } \\
\text { Difference }\end{array}$} & \multirow{2}{*}{$\begin{array}{l}\text { Std. Error } \\
\text { Difference }\end{array}$} & \multicolumn{2}{|c|}{$\begin{array}{l}\text { 95\% Confidence Interval of the } \\
\text { Difference }\end{array}$} \\
\hline & & & & & & & & & Lower & Upper \\
\hline \% leave & $\begin{array}{l}\text { Equal variances } \\
\text { assumed }\end{array}$ & .048 & .827 & -2.678 & 319 & .008 & .02605 & .00973 & .04520 & -.00691 \\
\hline & $\begin{array}{l}\text { Equal variances not } \\
\text { assumed }\end{array}$ & & & -2.707 & 299.493 & .007 & .02605 & .00963 & -.04499 & -.00711 \\
\hline
\end{tabular}


Fuente: elaboración propia basada en las estimaciones del resultado por distrito realizadas por Chris Hanretty y en la compilación del Daily Mirror sobre la posición previa de los legisladores frente al referéndum. 